\title{
Modification of PLGA Nanofibrous Mats by Electron Beam Irradiation for Soft Tissue Regeneration
}

\author{
Jae Baek Lee, ${ }^{1}$ Young-Gwang Ko, ${ }^{1}$ Donghwan Cho, ${ }^{1}$ Won Ho Park, ${ }^{2}$ Byeong Nam Kim, \\ Byeong Cheol Lee, ${ }^{3}$ Inn-Kyu Kang, ${ }^{4}$ and Oh Hyeong Kwon ${ }^{1}$ \\ ${ }^{1}$ Department of Polymer Science and Engineering, Kumoh National Institute of Technology, 1 Yangho-dong, \\ Gumi 730-701, Republic of Korea \\ ${ }^{2}$ Department of Advanced Organic Materials and Textile System Engineering, Chungnam National University, 99 Daehak-ro, \\ Yuseong-gu, Daejeon 305-764, Republic of Korea \\ ${ }^{3}$ Korea Atomic Energy Research Institute, 989 Daedeok-daero, Yuseong-gu, Daejeon 305-353, Republic of Korea \\ ${ }^{4}$ Department of Polymer Science and Engineering, Kyungpook National University, 80 Daehakro, Buk-gu, \\ Daegu 702-701, Republic of Korea
}

Correspondence should be addressed to Oh Hyeong Kwon; ohkwon@kumoh.ac.kr

Received 10 December 2014; Revised 19 February 2015; Accepted 11 March 2015

Academic Editor: Ilaria Armentano

Copyright (C) 2015 Jae Baek Lee et al. This is an open access article distributed under the Creative Commons Attribution License, which permits unrestricted use, distribution, and reproduction in any medium, provided the original work is properly cited.

\begin{abstract}
Biodegradable poly(lactide-co-glycolide) (PLGA) has found widespread use in modern medical practice. However, the degradation rate of PLGA should be adjusted for specific biomedical applications such as tissue engineering, drug delivery, and surgical implantation. This study focused on the effect of electron beam radiation on nanofibrous PLGA mats in terms of physical properties and degradation behavior with cell proliferation. PLGA nanofiber mats were prepared by electrospinning, and electron beam was irradiated at doses of 50, 100, 150, 200, 250, and $300 \mathrm{kGy}$. PLGA mats showed dimensional integrity after electron beam irradiation without change of fiber diameter. The degradation behavior of a control PLGA nanofiber ( $0 \mathrm{kGy})$ and electron beamirradiated PLGA nanofibers was analyzed by measuring the molecular weight, weight loss, change of chemical structure, and fibrous morphology. The molecular weight of the PLGA nanofibers decreased with increasing electron beam radiation dose. The mechanical properties of the PLGA nanofibrous mats were decreased with increasing electron beam irradiation dose. Cell proliferation behavior on all electron beam irradiated PLGA mats was similar to the control PLGA mats. Electron beam irradiation of PLGA nanofibrous mats is a potentially useful approach for modulating the biodegradation rate of tissue-specific nonwoven nanofibrous scaffolds, specifically for soft tissue engineering applications.
\end{abstract}

\section{Introduction}

Poly(lactide-co-glycolide) (PLGA) containing lactic and glycolic acid is a well known polymer approved by the United States Food and Drug Administration (FDA) for medical devices because of its biocompatibility, biodegradability, nontoxicity, and excellent mechanical properties [1]. The polymer has been extensively studied in a variety of biomedical applications such as drug delivery [2-5], tissue engineering [6-8], and surgical implantation [9-11]. Specifically, PLGA nanofibers fabricated by electrospinning have a highly interconnected porous network and an extremely large surface area-to-volume ratio (specific surface area). Therefore, PLGA nanofibers have the potential to be utilized in wound dressings for skin regeneration, drug delivery carriers, pharmaceuticals, and extracellular matrix- (ECM-) mimicking scaffolds for tissue engineering [12-16].

It is well known that PLGA is hydrolytically unstable although it is insoluble in aqueous media [17]. The degradation mechanism of PLGA is hydrolytic attack on the ester bonds. Through hydrolytic attack, chain scission occurs randomly in the polymeric material, causing it to degrade into lactic and glycolic acids [17-20]. In addition, hydrolytic degradation byproducts (lactic acid and glycolic acid) enter into the citric acid cycle and are metabolized and ultimately eliminated as carbon dioxide and water [21-23]. 
One of the most effective treatments for altering the physical and surface properties of bulk PLGA materials such as nonwoven mats, films, and molding is radiation [21, 24, 25]. Generally, radiation promotes polymerization (cross-linking) and/or degradation (main chain scission) [26, 27]. The polymerization process increases the molecular weight in consequence of cross-linking one main chain or side group to others. On the other hand, the degradation process occurs when chain scission of a polymer results in a reduction in the molecular weight [28]. These two processes are influenced by several factors including the chemical structure of the polymer, the dosage and dosage range of radiation, the environment, and the heat generated during irradiation [29-31].

The hydrolytic degradation behavior of the biodegradable polymer PLGA has been widely studied in various conditions, but controlling the degradation rate, especially in the case of a nanofibrous structure, remains to be an obstacle. It is well known that PLGA with a lactic and glycolic acid ratio of $50: 50$ is hydrolyzed much faster compared to other ratios (approximately 2 months) [18, 32]. However, the soft tissue scaffold, especially for skin tissue, should initially promote cell proliferation and then degrade approximately to the time required for wound healing (approximately 3 weeks). Thus, degradation kinetic for skin tissue regeneration must be adjusted from 3 to 4 weeks. Tunable biodegradation rates would allow for better tailoring of PLGA scaffolds for tissuespecific regeneration [33]. In this study, we fabricated PLGA nanofiber mats by electrospinning and subsequently electron beam irradiated with various doses and examined the influence of radiation on the molecular structure, physical properties, morphology, hydrolytic degradation behavior in vitro, and cell proliferation.

\section{Experimental}

2.1. Preparation of Nonwoven PLGA Mats. PLGA nanofibrous mats were fabricated using the electrospinning method. PLGA (50:50) was purchased from PURAC biochem (Netherlands) and 1,1,1,3,3,3-hexafluoro-2-propanol (HFIP) was obtained from Matrix scientific (Columbia, USA). The PLGA and HFIP were used without further purification. PLGA was dissolved in HFIP at a $6 \mathrm{wt} \%$ concentration by stirring overnight at room temperature. The polymer solution was placed into a syringe (Hamilton 81620 gastight syringe, USA) with a 21-gauge needle (Hamilton 91022 metal hub needle, USA). To create an electric field, a high voltage of $15 \mathrm{kV}$ was applied between the needle and the stainless steel drum collector (distance $12 \mathrm{~cm}$, drum diameter $23 \mathrm{~cm}$ ). The flow rate of the solution was $1 \mathrm{~mL} / \mathrm{h}$. The electrospun nonwoven PLGA nanofibrous mats were dried in a vacuum oven for $24 \mathrm{~h}$ at room temperature to remove any residual solvent.

2.2. Electron Beam Radiation. An electron beam (e-beam) was irradiated on the PLGA nanofibrous mats using the ELV8 as an Electron Beam Accelerator (EB Tech Co. Ltd., Korea). Irradiation of the electron beam onto the PLGA nanofibers was carried out in the air at room temperature. The accelerating voltage of the electron beam and beam current were
$1 \mathrm{MeV}$ and $17 \mathrm{~mA}$, respectively. The irradiation doses were 50, $100,150,200,250$, and $300 \mathrm{kGy}$. To avoid shrinkage and deformation of electrospun PLGA nanofibrous mats due to heat, samples were provided a 5 min break after every $25 \mathrm{kGy}$ dose of irradiation.

2.3. Measurement of Molecular Weight. Gel permeation chromatography (GPC) was used to determine the number average molecular weight $(\mathrm{Mn})$ and weight average molecular weight (Mw) of each e-beam irradiated PLGA nanofiber. GPC was performed with Alliance e2695 (Waters, USA) at $40^{\circ} \mathrm{C}$ using a refractive index detector. The flow rate was $1 \mathrm{~mL} / \mathrm{min}$ and tetrahydrofuran was used as the solvent. Polystyrene standards were used to calibrate the GPC.

2.4. Biodegradation Test. Each electron beam irradiated PLGA fiber was cut into a rectangular shape with dimensions of $20 \mathrm{~mm} \times 10 \mathrm{~mm}$ and sealed in a conical tube containing $10 \mathrm{~mL}$ of phosphate buffered saline (PBS, pH 7.4) after weighing the conical tube (weight of an empty conical tube, $m_{c}$ ) and samples (initial weight of nanofiber, $m_{i}$ ). The tubes were placed into a shaking water bath $\left(37^{\circ} \mathrm{C}\right)$ for various periods. After each designated period, PBS was removed and the tubes were dried in a vacuum oven at room temperature for 5 days; thereafter, the dry weight was measured (conical tube including nanofiber weight after degradation, $m_{f}$ ). The percentage of weight loss was calculated using the following equation:

$$
\frac{m_{i}-\left(m_{f}-m_{c}\right)}{m_{i}} \times 100=\text { wight } \operatorname{loss}(\%)
$$

where $m_{i}$ is the initial weight of the nanofiber, $m_{f}$ is the weight of the conical tube including the nanofiber after degradation, and $m_{c}$ is the weight of the empty conical tube.

2.5. Scanning Electron Microscopy. The morphological structure of the electrospun PLGA nanofibers after electron beam irradiation and a series of biodegradation tests was observed using a scanning electron microscope (SEM, JSM-6380, JEOL, Japan) at an accelerating voltage of 10-15 kV. Samples were sputter coated with platinum prior to observation. The diameter of the electrospun nanofibers was determined using image analysis software (IMT i-solution, Image \& Microscope Technology Inc.). Three representative images of each specimen were used for measuring the fiber diameters at 100 sites.

2.6. FTIR Spectroscopy. The intensity of the hydroxyl group in PLGA following electron beam irradiation and in vitro degradation was confirmed using an Attenuated Total Reflection- (ATR-) FTIR spectrophotometer (Vertex 80v, Bruker, USA). All spectra were obtained in a vacuum with a resolution value of $4 \mathrm{~cm}^{-1}$ by 256 scans in the wavenumber range of $800-4000 \mathrm{~cm}^{-1}$. Spectra data were modified with a linear baseline using spectrum software (OPUS 6.5, USA). 
2.7. Mechanical Properties. A universal testing machine (Instron 4467, Instron, USA) with a $100 \mathrm{~N}$ load cell was used to measure the change in mechanical properties (tensile stress and strain) of PLGA nanofibrous mats as a function of electron beam radiation dose. The specimens were cut into strips (dimension: $70.00 \times 6.00 \times 0.14 \mathrm{~mm}$ ) for testing by using a gauge length of $33 \mathrm{~mm}$. An extension rate of $20 \mathrm{~mm} / \mathrm{min}$ was applied. The test was performed 3 times.

2.8. Hydrophilicity and Shrinkage Tests. The water contact angle (Phoenix 300, SEO Co. Ltd., Korea) was measured to check the hydrophilicity of the electron beam irradiated PLGA nanofibrous mats by using the sessile drop method. The test was performed at room temperature and the contact angle was obtained $3 \mathrm{~s}$ after the deionized water was dropped. The measurements were performed 5 times.

For the shrinkage test, the electron beam irradiated PLGA nanofibrous mats were cut into $23 \mathrm{~mm}$ diameter discs. Each sample was immersed in PBS (pH 7.4) until sufficient contact with medium and dried at room temperature for $6 \mathrm{~h}$. The area of the dried samples was compared to the initial area.

2.9. Cell Viability. A colorimetric assay was used to measure cell viability with WST-1 reagent. Samples were cut into $23 \mathrm{~mm}$ discs, and each side was exposed to UV light for $12 \mathrm{~h}$. Samples were placed in 12-well plates and a glass ring was used to prevent them from floating. Cultured cells (NIH 3T3 fibroblasts) were seeded onto the samples at a density of $3.0 \times$ $10^{4}$ cells/scaffold with $1 \mathrm{~mL}$ of Dulbecco's Modified Eagle Medium (DMEM) and incubated for 7 days at $37^{\circ} \mathrm{C}$ in $5 \%$ $\mathrm{CO}_{2}$. The culture medium was changed every 2 days. After 7 days, culture medium was removed and $1 \mathrm{~mL}$ of a solution containing a 1:9 ratio of WST-1 reagent and DMEM was put into each well. Subsequently, samples were incubated for $4 \mathrm{~h}$ at $37^{\circ} \mathrm{C}$, and then $100 \mu \mathrm{L}$ of the incubated solution was collected and transferred to a 96-well plate. An ELISA reader (multiwell microplate reader) was used to detect the optical density of formazan at $450 \mathrm{~nm}$.

2.10. Histological Analysis. The pore size of a fibrous scaffold is directly proportional to the average diameter of fibers. Large pores in a scaffold promote cell infiltration. To facilitate cell infiltration into a scaffold interior, we fabricated another PLGA mat with average diameter of $1.56 \pm 0.16 \mu \mathrm{m}$ by increasing concentration PLGA solution. PLGA was dissolved in HFIP at an $8.5 \mathrm{wt} \%$ concentration and stirred overnight at room temperature. The scaffold was fabricated by electrospinning at a voltage of $15 \mathrm{kV}$, distance of $12 \mathrm{~cm}$, and flow rate of $1 \mathrm{~mL} / \mathrm{h}$. After electrospinning, the fabricated scaffold was dried in a vacuum oven at room temperature for $24 \mathrm{~h}$ to remove residual solvent.

Scaffolds sterilized with UV light were seeded with fibroblasts at a density of $3 \times 10^{5}$ cells/scaffold in $1 \mathrm{~mL}$ of DMEM. After $4 \mathrm{~h}$, scaffolds were washed with DMEM. Then the scaffolds were incubated at $37^{\circ} \mathrm{C}$ in $5 \% \mathrm{CO}_{2}$. After the fibroblasts were cultured in the PLGA mats for 1 and 2 weeks, the constructs were washed with PBS and fixed in $4 \%$ neutral buffered formalin for paraffin embedding and sectioning. The cell-seeded PLGA mat sections were stained with hematoxylin and eosin (HE) to visualize cellular infiltration. They were then mounted and analyzed with an optical microscope (Nikon).

2.11. Statistical Analysis. Statistical analysis was performed using data analysis software (KyPlot version 2.0, KyensLab, Inc.). Each value represented mean \pm standard deviation. Significance levels were determined by parametric Student's $t$-test and one-way ANOVA (analysis of variance) with a post hoc test by Tukey's method. Statistical significance was considered as $P<0.05$.

\section{Results and Discussion}

3.1. Morphology of E-Beam Irradiated Electrospun PLGA Mats. Randomly orientated nanofibrous PLGA mats were fabricated by electrospinning. The microstructure of the PLGA nanofibers is shown in Figure 1(a). The nanofibers consisted of a relatively uniform cylindrical shape with an average diameter of $735 \pm 107 \mathrm{~nm}$. The nanofibrous structure showed different sizes of pores and three-dimensional networks. PLGA nanofiber mats that were e-beam irradiated with 50, $100,150,200,250$, and $300 \mathrm{kGy}$ had negligible morphological differences (Figures $1(\mathrm{~b})-1(\mathrm{~g})$ ) with average fiber diameters of $767 \pm 113,760 \pm 108,771 \pm 107,765 \pm 112,768 \pm 119$, and $742 \pm 130 \mathrm{~nm}$, respectively. There was no significant difference among the average diameters of the 7 types of PLGA mats. The result indicates that e-beam irradiation did not affect the fiber diameters of PLGA mats. But PLGA mats become more brittle with increasing e-beam radiation dose. Particularly, PLGA mats treated with radiation doses over $200 \mathrm{kGy}$ required special care to prevent cracking.

3.2. Molecular Weight. The number average molecular weight $(\mathrm{Mn})$ and weight average molecular weight $(\mathrm{Mw})$ are plotted against the e-beam irradiation dose in Figure 2. Both $\mathrm{Mn}$ and $\mathrm{Mw}$ were dramatically decreased with increasing e-beam irradiation dose. There was a rapid reduction in both $\mathrm{Mn}$ and $\mathrm{Mw}$ with increasing irradiation dose up until $100 \mathrm{kGy}$. After $150 \mathrm{kGy}$, there is a slight reduction in both $\mathrm{Mn}$ and $\mathrm{Mw}$ with increasing dose. The results indicate that ebeam irradiation promoted degradation rather than polymerization. The high energy generated from the e-beam and the formation of peroxyl free radicals is responsible for the main chain-scission process, which resulted in splitting of the PLGA molecular chains [30].

3.3. Weight Loss and Morphological Deformation. As the ebeam radiation dose increased, the electrospun PLGA nanofibrous mats increased in brittleness throughout the biodegradation test in PBS. Control PLGA mats that did not undergo e-beam treatment were flexible until the 6th week of the degradation experiment. In contrast, the PLGA mat that received a radiation dose of $150 \mathrm{kGy}$ became brittle after 1 week of incubation. The nanofibrous mat administered a radiation dose of $100 \mathrm{kGy}$ became brittle after 2 weeks. With 


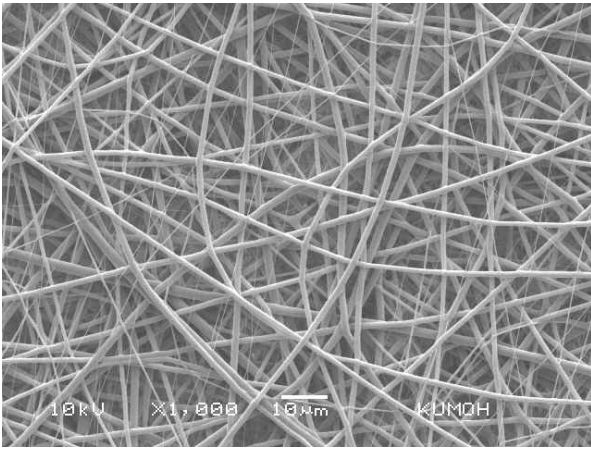

(a)

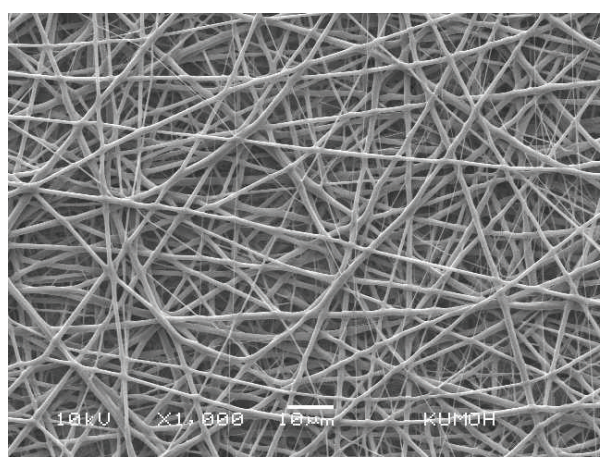

(c)

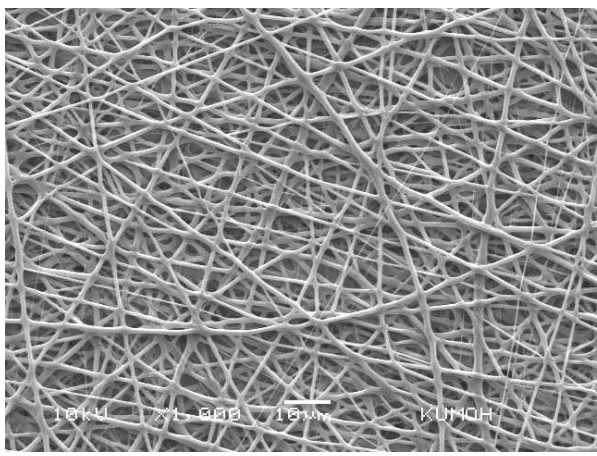

(e)

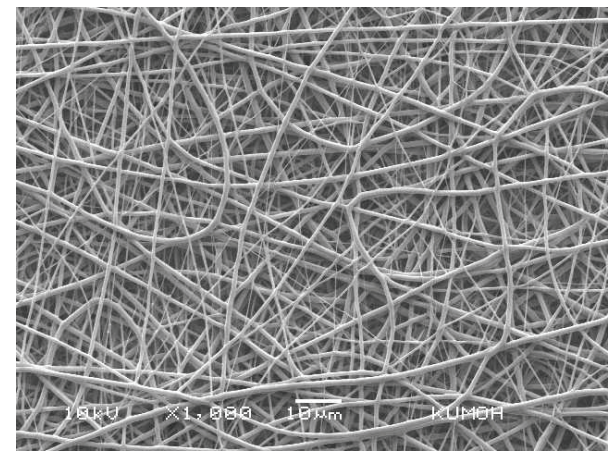

(b)

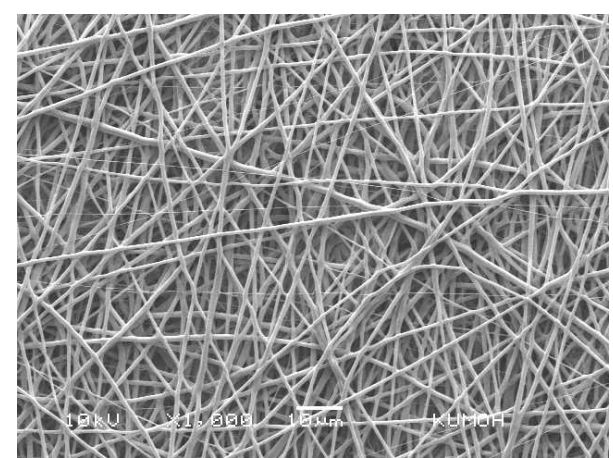

(d)

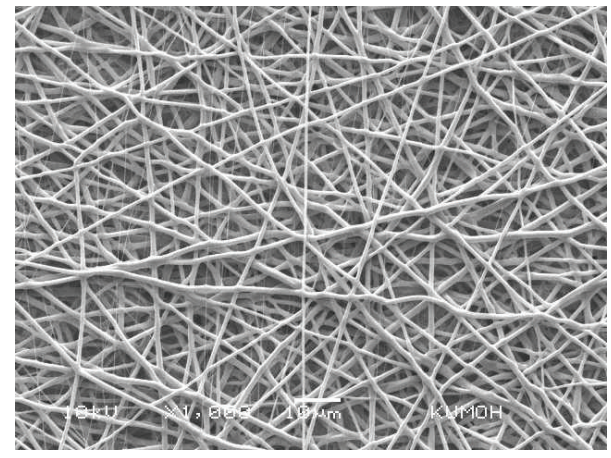

(f)

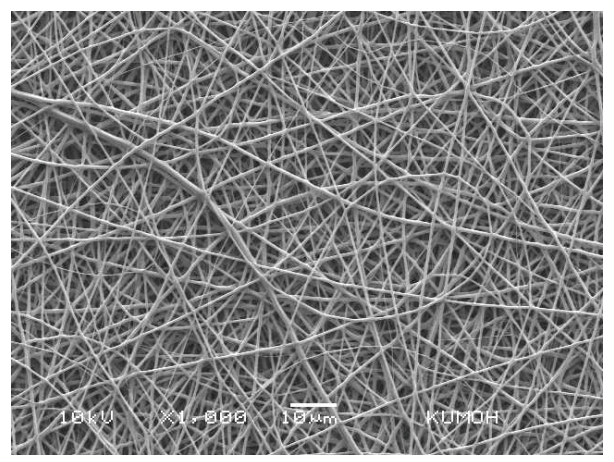

(g)

FIGURE 1: SEM images of the (a) control PLGA nanofibers and PLGA nanofibers e-beam irradiated with doses of (b) 50, (c) 100, (d) 150, (e) 200, (f) 250, and (g) $300 \mathrm{kGy}$. The magnification of the photomicrographs is 1,000x. 


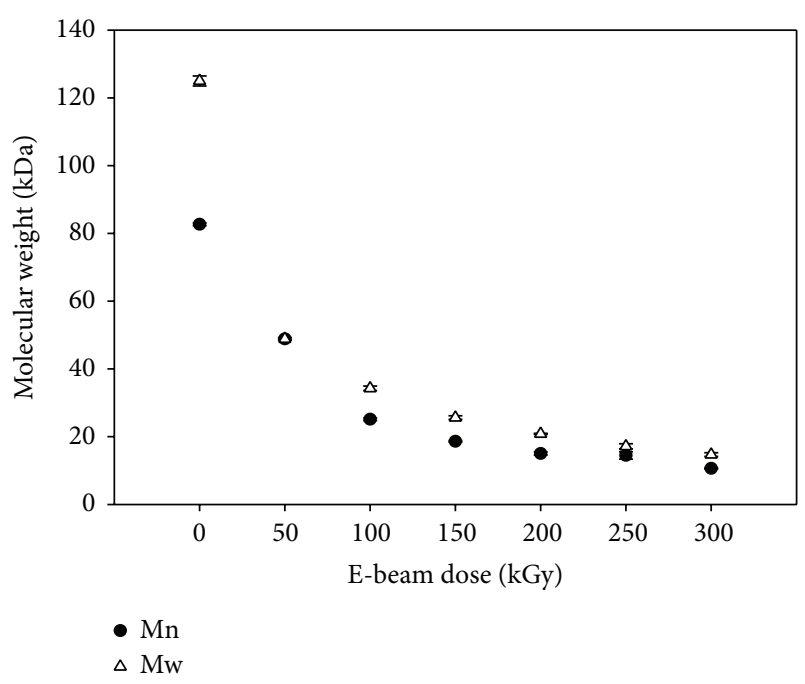

Figure 2: Number average (Mn) and weight average (Mw) molecular weight of e-beam irradiated PLGA nanofibers.

increasing radiation dose (200-300 kGy), the period before becoming fragile was dramatically shortened.

To observe changes in the biodegradation behavior in response to e-beam treatment, the weight loss of each PLGA mat was measured as a function of incubation time in PBS (Figure 3). Throughout the 7-week incubation period, samples exposed to higher radiation doses degraded more quickly. PLGA nanofibrous mats irradiated with more than $150 \mathrm{kGy}$ lost more than $80 \%$ of their initial weight after 6 weeks of incubation, while control PLGA mats without ebeam treatment exhibited a weight loss of only $10 \%$. Nonirradiated electrospun PLGA nanofibers exhibited little weight loss until the 6th week of incubation, when the rate of degradation suddenly increased.

Samples irradiated with doses of 50, 100, and $150 \mathrm{kGy}$ showed dramatically increased rates of degradation after 4, 3, and 2 weeks, respectively. Moreover, PLGA nanofibrous mats irradiated with more than $200 \mathrm{kGy}$ decreased in weight rapidly after the first week of incubation. These results were consistent with the morphological analysis (Figure 4), which showed a faster change in the PLGA nanofiber morphology to a film state with increasing radiation dose. Nonirradiated PLGA nanofibers maintained their original nanofibrous morphology until 6 weeks of incubation, whereas e-beam irradiated nanofibrous PLGA mats lost their fibrous shape and became film-like mats without pores. The morphological collapse from fibers to film became gradually faster with increasing radiation dose. The incubation time to morphological collapse closely corresponded to the time to increased degradation.

In the initial stages of degradation, PLGA exhibits little weight loss. This stage is called the induction period. After this period, the weight loss is accelerated and the degradation rate is increased [34]. According to Raghuvanshi, the induction period is the first stage that involves random chain scission, where no appreciable weight loss occurs, but the molecular weight of the polymer decreases considerably [35].

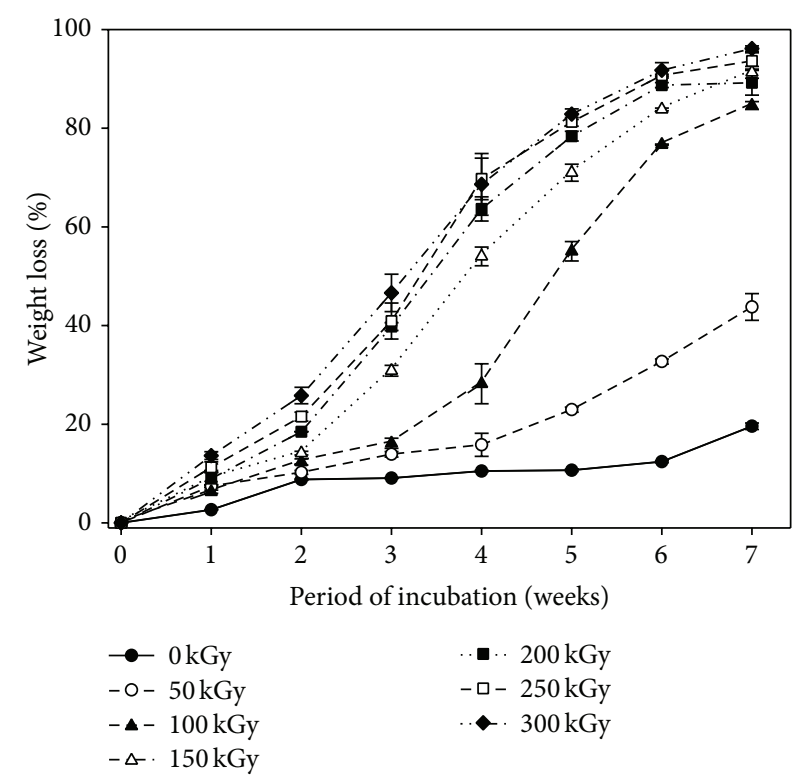

FIgURE 3: Weight loss of e-beam irradiated PLGA nanofibers as a function of length of incubation in $\mathrm{PBS}\left(37^{\circ} \mathrm{C}\right)$ over a period of 7 weeks. Data are expressed as the mean \pm S.D. of 3 samples.

This is the reason why e-beam irradiated PLGA nanofibers degraded much faster than nonirradiated PLGA. It has been reported that the degradation rate of a polymer is affected by its molecular weight [36]. As shown in Figures 3 and 4, the molecular weight of PLGA is significantly decreased when the main chain is ruptured by e-beam irradiation, so the induction period is skipped, and rapid weight loss begins when oligomers (soluble fragments) and monomers (glycolic acid, lactic acid) are formed in a short period.

3.4. ATR-FTIR Analysis. The ATR-FTIR spectra of PLGA nanofiber mats after 2 weeks of incubation are shown in Figure 5(a). All spectra exhibit $\mathrm{C}=\mathrm{O}$ stretching around $1750 \mathrm{~cm}^{-1}$, C-O bands in the $1085-1450 \mathrm{~cm}^{-1}$ regions indicating the presence of an ester group and bands at 2995, 2948, and $2881 \mathrm{~cm}^{-1}$ due to alkyl groups. Similar peaks have been recorded for PLGA nanofibers [37, 38]. But we recognized an unidentified peak around $1600 \mathrm{~cm}^{-1}$ that increases transmittance intensity according to radiation dose. These peaks appeared after degradation test of e-beam irradiated PLGA. We assumed that the peak around $1600 \mathrm{~cm}^{-1}$ to carboxylic acid salts $(\mathrm{O}=\mathrm{C}-\mathrm{ONa})$ asymmetric stretching is induced from mixture of acidic oligomers of PLGA and PBS, because the intensity of a transmittance of functional group may be affected by solvent during biodegradation test as well [39]. On the other hand, there was a distinctive difference in the ATRFTIR spectra between nonirradiated and irradiated PLGA nanofibers. The control PLGA mats showed negligible O-H group intensity. Upon irradiation, all the irradiated PLGA nanofibers showed a broad peak of $\mathrm{O}-\mathrm{H}$ stretching from 3100 to $3600 \mathrm{~cm}^{-1}$ and the intensity increased with increasing radiation dose. The $\mathrm{O}-\mathrm{H}$ stretching peak indicates the formation of the hydroxyl group during degradation (Figure 5(b)). 


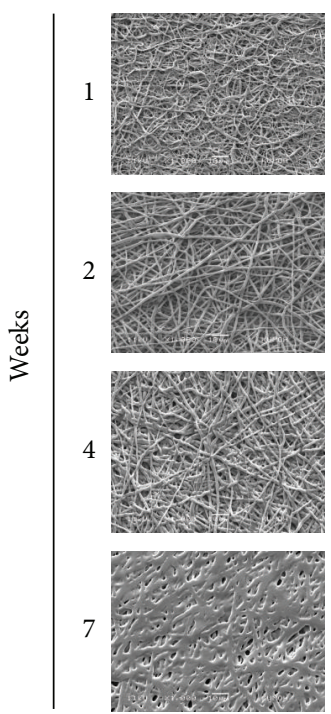

(a)
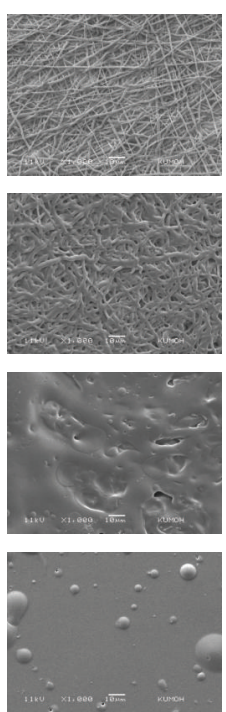

(b)
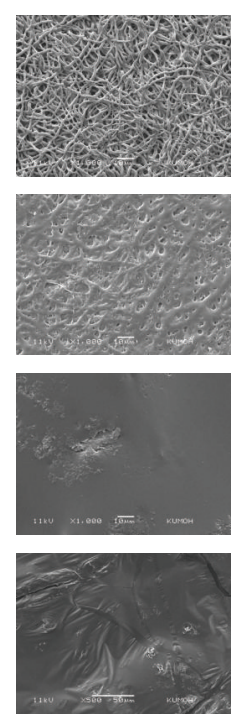

(c)
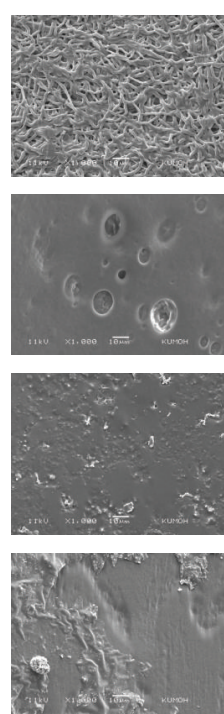

(d)
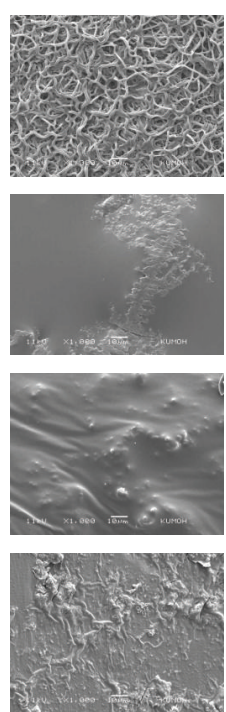

(e)
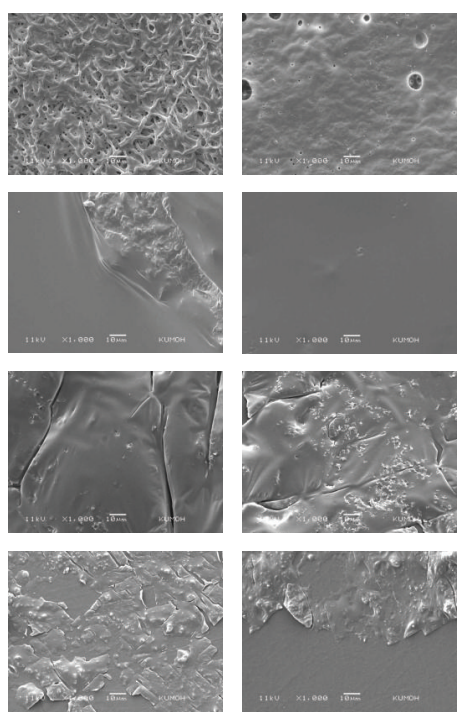

(f)
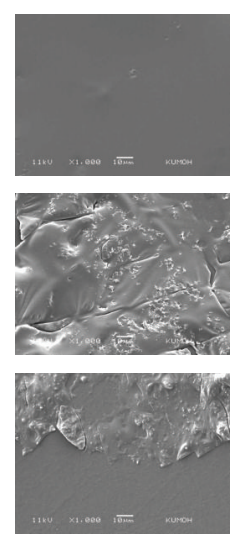

(g)

FIGURE 4: Morphology of e-beam irradiated PLGA nanofiber mats treated with doses of (a) 0, (b) 50, (c) 100, (d) 150, (e) 200, (f) 250, and (g) $300 \mathrm{kGy}$ over 7 weeks in $\mathrm{PBS}\left(37^{\circ} \mathrm{C}\right)$. The magnification of the photomicrographs is $1,000 \mathrm{x}$.

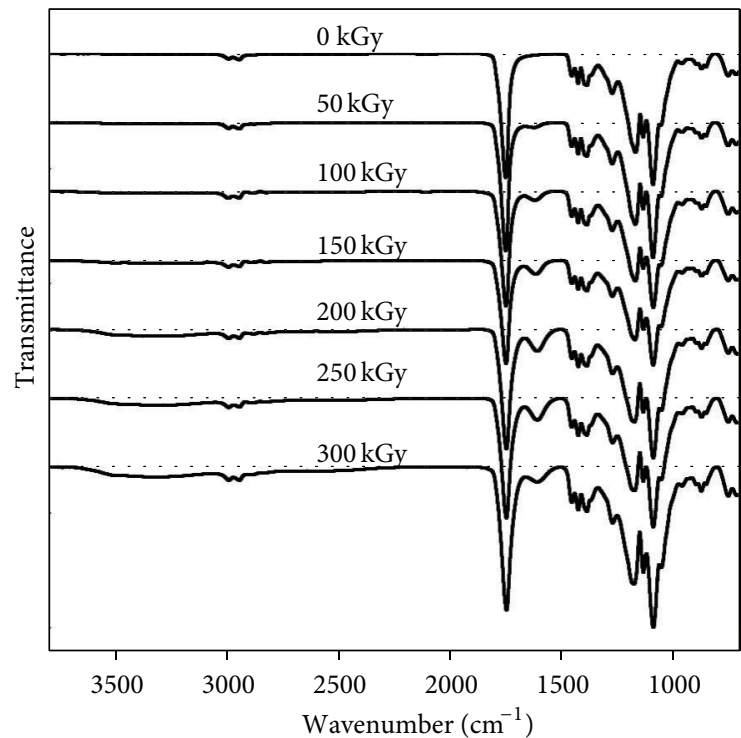

(a)

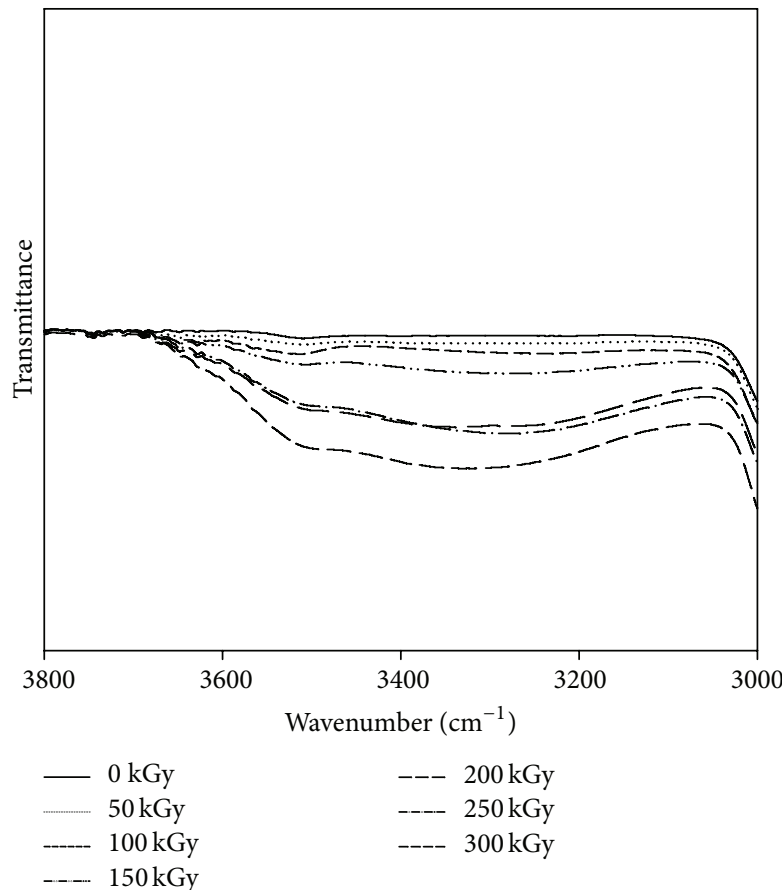

(b)

FIGURE 5: ATR-FTIR spectra for each e-beam irradiated (0-300 kGy) PLGA nanofiber mats after 2 weeks of incubation in PBS. Scan area of $4000-800 \mathrm{~cm}^{-1}$ wavenumber range (a) and magnified spectra for identification of hydroxyl group ratio around $3600-3100 \mathrm{~cm}^{-1}$ wavenumber range (b).

In aqueous conditions, a hydroxyl group was formed due to hydrolysis of the ester group of PLGA. The O-H stretching peak formation indicates that hydrolytic degradation occurred in PBS during incubation, and the increase in peak intensity with increasing radiation dose indicates that PLGA nanofibers irradiated with higher doses degrade more quickly. As mentioned above, low-molecular-weight PLGA does not exhibit an induction period and it quickly undergoes weight loss.

3.5. Mechanical Properties. Because PLGA has excellent physical properties among the synthetic biodegradable polymers, changes to the mechanical characteristics of PLGA 
TABLE 1: Mechanical properties of e-beam irradiated PLGA nanofiber mats as a function of radiation dose.

\begin{tabular}{lccc}
\hline $\begin{array}{l}\text { E-beam dose } \\
(\mathrm{kGy})\end{array}$ & $\begin{array}{c}\text { Modulus } \\
(\mathrm{MPa})\end{array}$ & $\begin{array}{c}\text { Tensile stress } \\
(\mathrm{MPa})\end{array}$ & $\begin{array}{c}\text { Elongation at } \\
\text { break }(\%)\end{array}$ \\
\hline 0 & $260 \pm 7$ & $10.0 \pm 0.7$ & $345 \pm 10$ \\
50 & $226 \pm 21$ & $7.1 \pm 0.3$ & $274 \pm 25$ \\
100 & $210 \pm 14$ & $5.7 \pm 0.3$ & $194 \pm 21$ \\
150 & $182 \pm 18$ & $5.8 \pm 0.8$ & $8 \pm 1$ \\
\hline
\end{tabular}

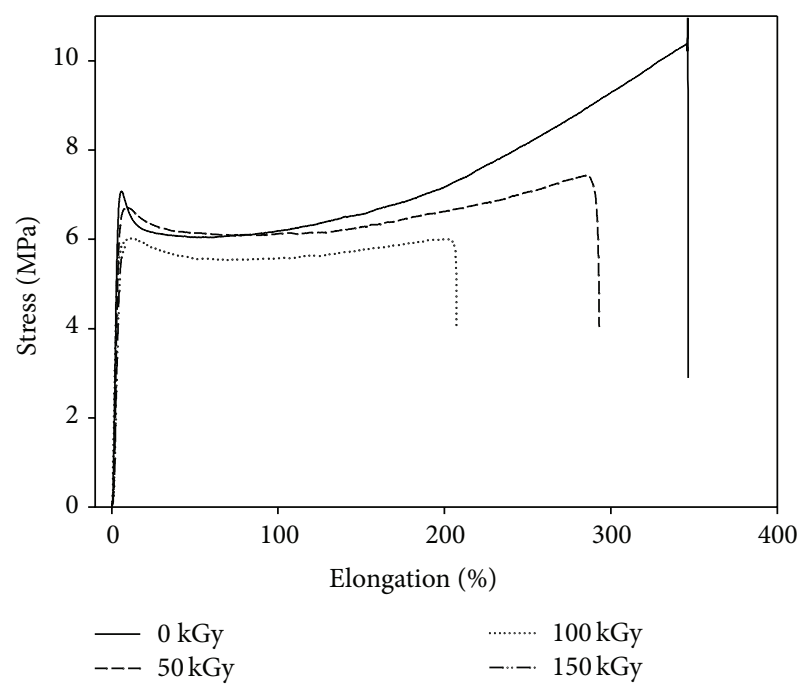

FIGURE 6: Stress-strain curves of e-beam irradiated PLGA nanofiber mats.

nanofibrous mats because of e-beam treatment were measured by analyzing the tensile stress-strain curves (Figure 6). The test was performed on PLGA mats irradiated with ebeam doses ranging from 0 to $150 \mathrm{kGy}$. Specimens treated with $200 \mathrm{kGy}$ were too weak to test and broke during gripping. The nonirradiated PLGA mats exhibited higher mechanical properties (modulus of $260 \mathrm{MPa}$, elongation percentage of $345 \%$, and $10 \mathrm{MPa}$ of tensile strength) when compared with the e-beam irradiated PLGA mats, and the mechanical properties decreased with increasing radiation dose (Table 1). Notably, the percent elongation at failure of the PLGA mat irradiated with $150 \mathrm{kGy}$ was drastically decreased.

The increase in brittleness of the polymer is caused by a decrease in molecular weight. As entanglements are formed through the formation of chains looping around one another, the number of entanglement points increases as the polymer chain lengthens [40]. In this experiment, the high molecular weight PLGA shifted to a lower molecular weight because of treatment with e-beam irradiation. Thus, the shortened main chain length caused a reduction in the number of entanglement points, and the mechanical properties of amorphous PLGA were decreased.

3.6. Hydrophilicity. Water contact angles were measured to check the surface hydrophilicity of electrospun PLGA mats in accordance with the e-beam radiation dose (Figure 7). The

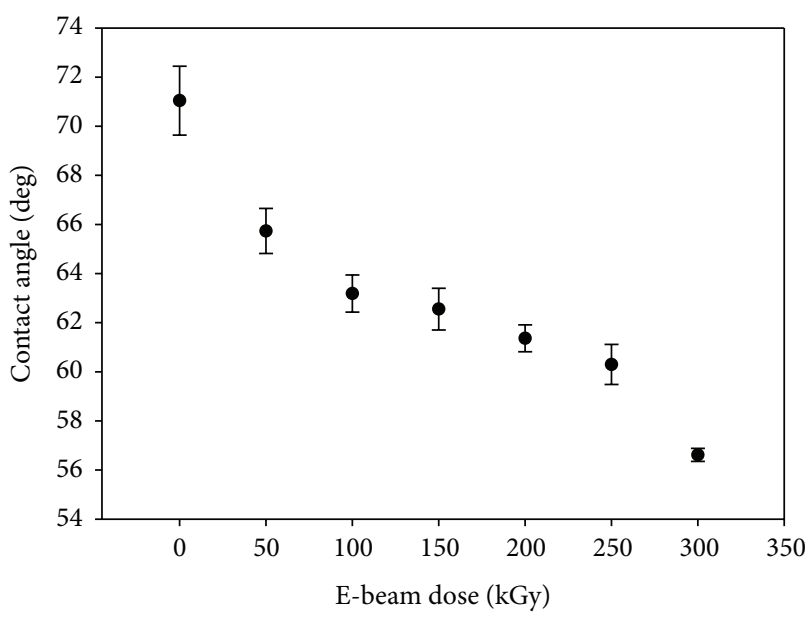

FIGURE 7: Water contact angles of PLGA mats administered each ebeam irradiation dose. Data are expressed as the mean \pm S.D. of 5 samples.

water contact angle of the nonirradiated PLGA mat was about $71 \pm 0.51^{\circ}$ and gradually decreased to $65 \pm 0.88^{\circ}, 64 \pm 1.11^{\circ}$, $62 \pm 1.05^{\circ}, 62 \pm 0.73^{\circ}, 61 \pm 0.87^{\circ}$, and $56 \pm 0.88^{\circ}$ at radiation doses of 50,100,150, 200, 250, and $300 \mathrm{kGy}$, respectively. The ATR-FTIR spectra of e-beam irradiated PLGA did not show hydroxyl groups (data not shown). Generally, e-beam irradiated PLGA forms alcohol groups caused by main chain scission [19], but these were not observed in the electrospun PLGA. These results indicate that the initial change in hydrophilicity of the electrospun PLGA is insignificant.

3.7. Shrinkage Test. Each e-beam irradiated PLGA nanofibrous mat was immersed in PBS to investigate the shrinkage behavior. The nonirradiated PLGA mats shrank during drying at room temperature (Supplementary data 1) (see data 1 in Supplementary Material available online at http://dx.doi.org/10.1155/2015/295807). In contrast, the ebeam irradiated PLGA (50-300 kGy) maintained its original size.

If an amorphous polymer has a low enough glass transition temperature $(\mathrm{Tg})$, the main chain of the aligned polymer will gradually become randomly coiled due to thermally induced relaxation [35]. PLGA is an easily shrinkable polymer because the $\mathrm{Tg}$ of PLGA is about $45-55^{\circ} \mathrm{C}$ (depending on the molecular weight). The Tg of wet PLGA nanofibers fabricated by electrospinning decreased to $31.4^{\circ} \mathrm{C}$, and this change in the thermal property contributed to the shrinkage behavior [41]. The $\mathrm{Tg}$ of the nonirradiated PLGA mat was determined as $31.4^{\circ} \mathrm{C}$ and gradually decreased to $26.0,25.5$, $25.2,25.2$, and $24.2^{\circ} \mathrm{C}$ at radiation doses of $50,100,150,200$, 250 , and $300 \mathrm{kGy}$, respectively. According to the random walk model, a polymer chain has a greater chance of being curled in a spherical shape and less of a chance of being stretched out. If each conformation has the same probability or statistical weight, polymer chains tend to be entangled in a globular shape as opposed to a linear shape [42]. When an extended polymer is relaxed to a random coil at a temperature above 


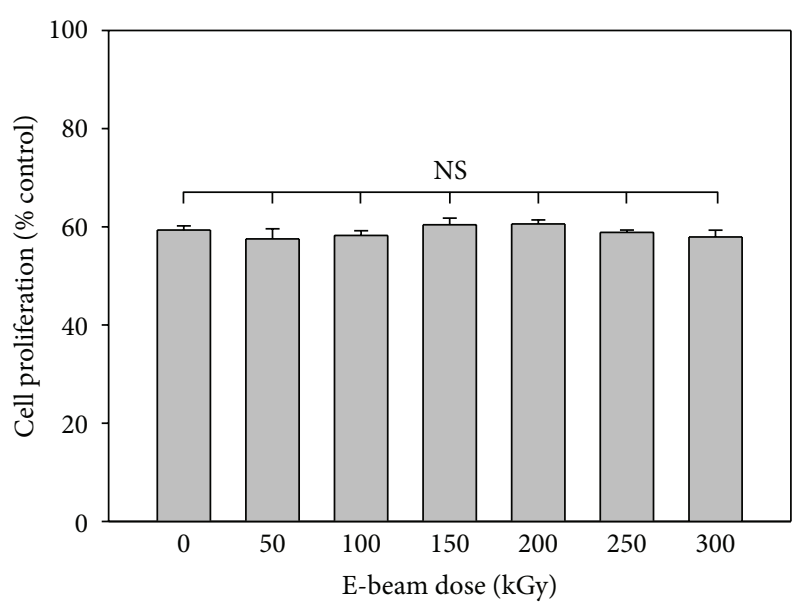

FIGURE 8: Cell proliferation evaluated by WST-1 assay of each ebeam irradiated PLGA nanofibrous mat after 7 days. Data are expressed as the mean \pm S.D. of 3 samples. NS means not significant $(P>0.05)$.

$\mathrm{Tg}$, short chains form relatively smaller spherical shapes than long chains, but the small spheres are connected. Electrospun PLGA is fabricated in an electric field, so the PLGA solution is pulled resulting in the fiber having a more extended structure than normal PLGA. Therefore, the nonirradiated electrospun PLGA, which had a long polymer chain, was relaxed above the Tg resulting in shrinkage. On the other hand, the PLGA that was ruptured by e-beam irradiation exhibited no shrinkage.

3.8. Cell Viability and Proliferation in the PLGA Scaffolds. Cell viability after 7 days on the nonirradiated and irradiated PLGA mats is shown in Figure 8. The viability of the cells on the nonirradiated PLGA nanofibrous mat was about $61 \%$ when compared to tissue culture polystyrene. The cell viability on PLGA nanofibers irradiated with 50, 100, 150, 200, 250 , and $300 \mathrm{kGy}$ was $55,58,63,60,58$, and $57 \%$, respectively. All of the PLGA (nonirradiated and irradiated) showed similar cell viability regardless of the changes in surface and mechanical properties. Based on these results, e-beam irradiation of PLGA nanofibrous mats has no adverse effect on cell viability.

Histological images of the cell-seeded PLGA mats after 7 and 14 days of culture are shown in Figure 9. The histological images show that cultured cells migrated from the surface of the scaffold into the inner part of the electrospun PLGA and proliferated very well after 2 weeks of culture. As shown in Figure 9, the fibrous structure gradually disappeared after 2 weeks of incubation, and the degraded space was spontaneously occupied by cells.

\section{Conclusions}

PLGA nanofibrous mats were fabricated using the electrospinning method. Electrospun PLGA mats were treated with electron beam radiation without morphological deformation of the nanofibrous structure (50-300 kGy). The molecular

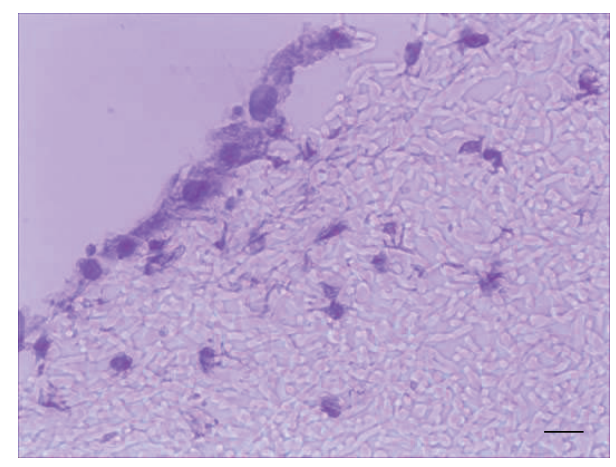

(a)

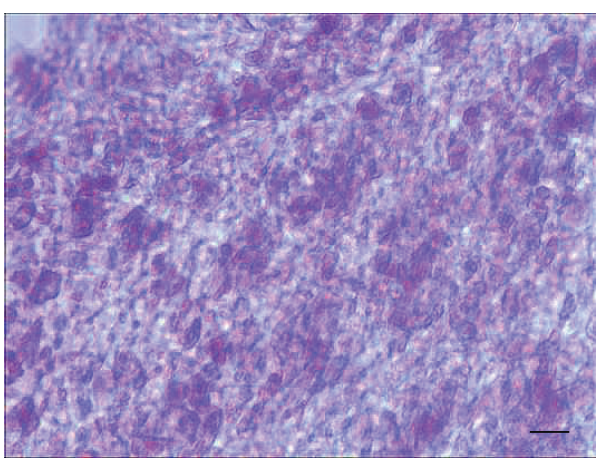

(b)

FIGURE 9: Hematoxylin and eosin staining of cells in a $150 \mathrm{kGy}$ ebeam irradiated PLGA nanofibrous scaffold (cross section) after a culture period of (a) 1 week and (b) 2 weeks. Scale bars $=10 \mu \mathrm{m}$.

weight and mechanical properties of PLGA nanofibers were decreased by e-beam radiation treatment due to the main chain scission of the PLGA backbone. Weight loss of the PLGA mats treated with $150-300 \mathrm{kGy}$ e-beam radiation was increased significantly compared to the mats treated with 50 and $100 \mathrm{kGy}$ after 2 weeks. The nanofibrous structure of the PLGA treated with 150-300 kGy e-beam radiation quickly collapsed after 2 weeks of incubation in PBS, while all of the e-beam treated PLGA mats exhibited no dimensional changes. Both the $\mathrm{OH}$ stretch peak intensity and surface hydrophilicity of PLGA mats were increased with increasing e-beam radiation dose. E-beam irradiation of PLGA had no effect on the proliferation of cells seeded on the nanofibrous mats. E-beam irradiation could be applied to adjust the biodegradation rate of the PLGA nanofibrous structure to allow for cell infiltration and homogeneous tissue proliferation. E-beam irradiated PLGA nanofibrous mats showed rapid biodegradation demonstrating their potential for soft tissue engineering applications, specifically skin tissue.

\section{Conflict of Interests}

The authors declare that there is no conflict of interests regarding the publication of this paper. 


\section{Acknowledgment}

This work was supported by the National Research Foundation of Korea (NRF) grant funded by the Korean government (MSIP) (NRF-2013M2B2A4040978 and NRF2012M2A2A6035747).

\section{References}

[1] J. M. Anderson and M. S. Shive, "Biodegradation and biocompatibility of PLA and PLGA microspheres," Advanced Drug Delivery Reviews, vol. 28, no. 1, pp. 5-24, 1997.

[2] R. A. Jain, "The manufacturing techniques of various drug loaded biodegradable poly(lactide-co-glycolide) (PLGA) devices," Biomaterials, vol. 21, no. 23, pp. 2475-2490, 2000.

[3] E.-R. Kenawy, G. L. Bowlin, K. Mansfield et al., "Release of tetracycline hydrochloride from electrospun poly(ethylene-covinylacetate), poly(lactic acid), and a blend," Journal of Controlled Release, vol. 81, no. 1-2, pp. 57-64, 2002.

[4] K. Kim, Y. K. Luu, C. Chang et al., "Incorporation and controlled release of a hydrophilic antibiotic using poly(lactide-coglycolide)-based electrospun nanofibrous scaffolds," Journal of Controlled Release, vol. 98, no. 1, pp. 47-56, 2004.

[5] H. Tamber, P. Johansen, H. P. Merkle, and B. Gander, "Formulation aspects of biodegradable polymeric microspheres for antigen delivery," Advanced Drug Delivery Reviews, vol. 57, no. 3, pp. 357-376, 2005.

[6] W.-J. Li, C. T. Laurencin, E. J. Caterson, R. S. Tuan, and F. K. Ko, "Electrospun nanofibrous structure: a novel scaffold for tissue engineering," Journal of Biomedical Materials Research, vol. 60, no. 4, pp. 613-621, 2002.

[7] X. H. Zong, H. Bien, C.-Y. Chung et al., "Electrospun finetextured scaffolds for heart tissue constructs," Biomaterials, vol. 26, no. 26, pp. 5330-5338, 2005.

[8] A. S. Badami, M. R. Kreke, M. S. Thompson, J. S. Riffle, and A. S. Goldstein, "Effect of fiber diameter on spreading, proliferation, and differentiation of osteoblastic cells on electrospun poly(lactic acid) substrates," Biomaterials, vol. 27, no. 4, pp. 596606, 2006.

[9] S. Li, H. Garreau, and M. Vert, "Structure-property relationships in the case of the degradation of massive poly $(\alpha$-hydroxy acid) in aqueous media; part 3: influence of the morphology of poly (l-lactic acid)," Journal of Materials Science: Materials in Medicine, vol. 1, pp. 198-206, 1990.

[10] S. M. Li, H. Garreau, and M. Vert, "Structure-property relationships in the case of the degradation of massive aliphatic poly- $(\alpha-$ hydroxy acids) in aqueous media-part 1: poly(dl-lactic acid)," Journal of Materials Science: Materials in Medicine, vol. 1, no. 3, pp. 123-130, 1990.

[11] S. M. Li, H. Garreau, and M. Vert, "Structure-property relationships in the case of the degradation of massive $\operatorname{poly}(\alpha-$ hydroxy acids) in aqueous media-part 2 Degradation of lactide-glycolide copolymers: PLA37.5GA25 and PLA75GA25," Journal of Materials Science: Materials in Medicine, vol. 1, no. 3, pp. 131-139, 1990.

[12] B. M. Min, Y. Y. Kim, J. M. Lee, and W. H. Park, "Formation of nanostructured poly(lactic-co-glycolic acid)/chitin matrix and its cellular response to normal human keratinocytes and fibroblasts," Carbohydrate Polymers, vol. 57, no. 3, pp. 285-292, 2004.
[13] Y. M. Shin, M. M. Hohman, M. P. Brenner, and G. C. Rutledge, "Experimental characterization of electrospinning: the electrically forced jet and instabilities," Polymer, vol. 42, no. 25, pp. 9955-9967, 2001.

[14] E. K. F. Yim, R. M. Reano, S. W. Pang, A. F. Yee, C. S. Chen, and K. W. Leong, "Nanopattern-induced changes in morphology and motility of smooth muscle cells," Biomaterials, vol. 26, no. 26, pp. 5405-5413, 2005.

[15] Y. Wan, Y. Wang, Z. Liu et al., "Adhesion and proliferation of OCT-1 osteoblast-like cells on micro- and nano-scale topography structured poly(L-lactide)," Biomaterials, vol. 26, no. 21, pp. 4453-4459, 2005.

[16] A.-S. Andersson, J. Brink, U. Lidberg, and D. S. Sutherland, "Influence of systematically varied nanoscale topography on the morphology of epithelial cells," IEEE Transactions on Nanobioscience, vol. 2, no. 2, pp. 49-57, 2003.

[17] L. G. Griffith, "Polymeric biomaterials," Acta Materialia, vol. 48, no. 1, pp. 263-277, 2000.

[18] J. P. Kitchell and D. L. Wise, "Poly(lactic/glycolic acid) biodegradable drug-polymer matrix systems," Methods in Enzymology, vol. 112, pp. 436-448, 1985.

[19] R. Jalil and J. R. Nixon, "Biodegradable poly(lactic acid) and poly(lactide-co-glycolide) microcapsules: problems associated with preparative techniques and release properties," Journal of Microencapsulation, vol. 7, no. 3, pp. 297-325, 1990.

[20] S. C. J. Loo, C. P. Ooi, and Y. C. F. Boey, "Radiation effects on poly(lactide-co-glycolide) (PLGA) and poly(L-lactide) (PLLA)," Polymer Degradation and Stability, vol. 83, no. 2, pp. 259-265, 2004.

[21] R. A. Jain, "The manufacturing techniques of various drug loaded biodegradable poly(lactide-co-glycolide) (PLGA) devices," Biomaterials, vol. 21, no. 23, pp. 2475-2490, 2000.

[22] I. Bala, S. Hariharan, and M. N. V. R. Kumar, "PLGA nanoparticles in drug delivery: the state of the art," Critical Reviews in Therapeutic Drug Carrier Systems, vol. 21, no. 5, pp. 387-422, 2004.

[23] T. R. Tice and D. R. Cowsar, "Biodegradable controlled-release parenteral systems," Pharmaceutical Technology, vol. 8, no. 11, pp. 26-35, 1984.

[24] N. Nagasawa, A. Kaneda, S. Kanazawa et al., "Application of poly(lactic acid) modified by radiation crosslinking," Nuclear Instruments and Methods in Physics Research Section B: Beam Interactions with Materials and Atoms, vol. 236, no. 1-4, pp. 611616, 2005.

[25] H. Mitomo, A. Kaneda, T. M. Quynh, N. Nagasawa, and F. Yoshii, "Improvement of heat stability of poly(l-lactic acid) by radiation-induced crosslinking," Polymer, vol. 46, no. 13, pp. 4695-4703, 2005.

[26] A. Charlesby, Radiation Chemistry Principles and Applications, VCH, New York, NY, USA, 1987.

[27] X. Zhang, M. Kotaki, S. Okubayashi, and S. Sukigara, "Effect of electron beam irradiation on the structure and properties of electrospun PLLA and PLLA/PDLA blend nanofibers," Acta Biomaterialia, vol. 6, no. 1, pp. 123-129, 2010.

[28] T. Ichikawa, "Mechanism of radiation-induced degradation of poly(methyl methacrylate)-temperature effect," Nuclear Instruments and Methods in Physics Research Section B: Beam Interactions with Materials and Atoms, vol. 105, no. 1-4, pp. 150153, 1995.

[29] A. Charlesby, Atomic Radiation and Polymers, Pergamon, Oxford, UK, 1960. 
[30] S. C. J. Loo, C. P. Ooi, and Y. C. F. Boey, "Influence of electronbeam radiation on the hydrolytic degradation behaviour of poly(lactide-co-glycolide) (PLGA)," Biomaterials, vol. 26, no. 18, pp. 3809-3817, 2005.

[31] C. M. Agrawal, D. Huang, J. P. Schmitz, and K. A. Athanasiou, "Elevated temperature degradation of a 50:50 copolymer of PLA-PGA," Tissue Engineering, vol. 3, no. 4, pp. 345-352, 1997.

[32] D. H. Lewis, M. Chasin, and R. Langer, Eds., Biodegradable Polymers as Drug Delivery System, Marcel Dekker, New York, NY, USA, 1990.

[33] R. Lanza, R. Langer, and J. Vacanti, Eds., Principles of Tissue Engineering, Elsevier Academic Press, Burlington, Mass, USA, 3rd edition, 2007.

[34] Y. You, B.-M. Min, S. J. Lee, T. S. Lee, and W. H. Park, "In vitro degradation behavior of electrospun polyglycolide, polylactide, and poly(lactide-co-glycolide)," Journal of Applied Polymer Science, vol. 95, no. 2, pp. 193-200, 2005.

[35] R. S. Raghuvanshi, M. Singh, and G. P. Talwar, "Biodegradable delivery system for single step immunization with tetanus toxoid," International Journal of Pharmaceutics, vol. 93, no. 1-3, pp. R1-R5, 1993.

[36] X. S. Wu, Encyclopedia Handbook of Biomaterials and Bioengineering, Marcel Dekker, New York, NY, USA, 1995.

[37] I. Armentano, M. Dottori, D. Puglia, and J. M. Kenny, "Effects of carbon nanotubes (CNTs) on the processing and in-vitro degradation of poly(dl-lactide-co-glycolide)/CNT films," Journal of Materials Science: Materials in Medicine, vol. 19, no. 6, pp. 23772387, 2008.

[38] M. V. Jose, V. Thomas, K. T. Johnson, D. R. Dean, and E. Nyairo, "Aligned PLGA/HA nanofibrous nanocomposite scaffolds for bone tissue engineering," Acta Biomaterialia, vol. 5, no. 1, pp. 305-315, 2009.

[39] G. Socrates, Infrared and Raman Characteristic Group Frequencies: Tables and Charts, Wiley, New York, NY, USA, 3rd edition, 2001.

[40] L. E. Nielsen and R. F. Landel, Mechanical Properties of Polymer and Composites, Marcel Dekker, New York, NY, USA, 1994.

[41] Y.-J. Liu, H.-L. Jiang, Y. Li, and K.-J. Zhu, "Control of dimensional stability and degradation rate in electrospun composite scaffolds composed of poly(D,L-lactide-co-glycolide) and poly(E-caprolactone)," Chinese Journal of Polymer Science (English Edition), vol. 26, no. 1, pp. 63-71, 2008.

[42] W. Feller, An Introduction to Probability Theory and Its Applications, Volume I, Wiley, New York, NY, USA, 1966. 

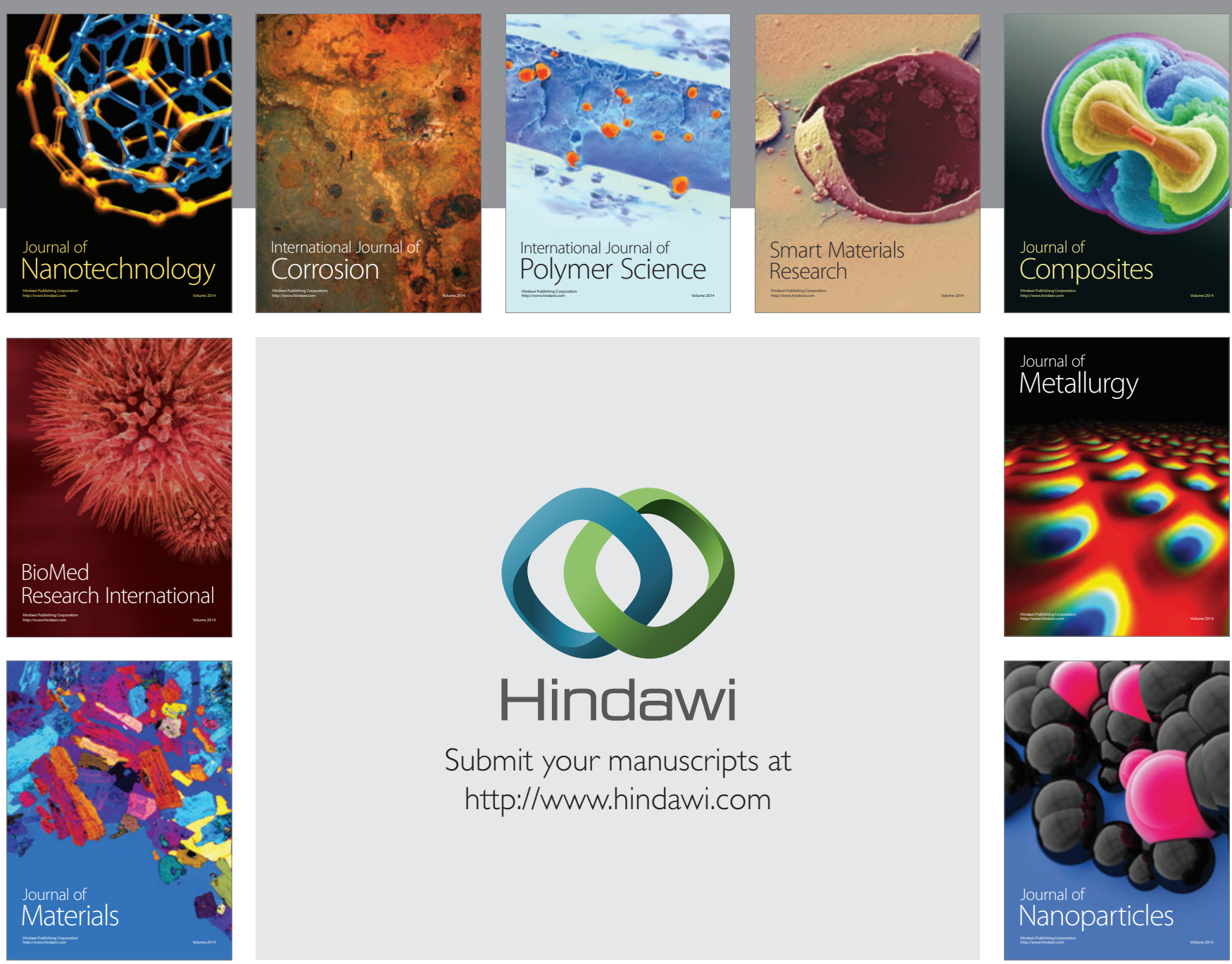

Submit your manuscripts at http://www.hindawi.com
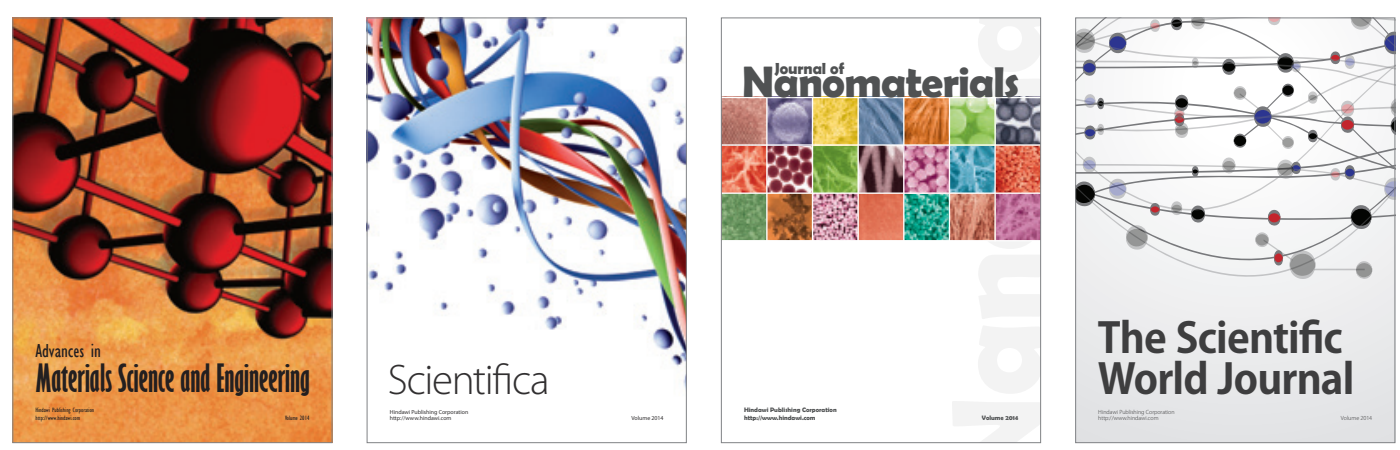

\section{The Scientific World Journal}
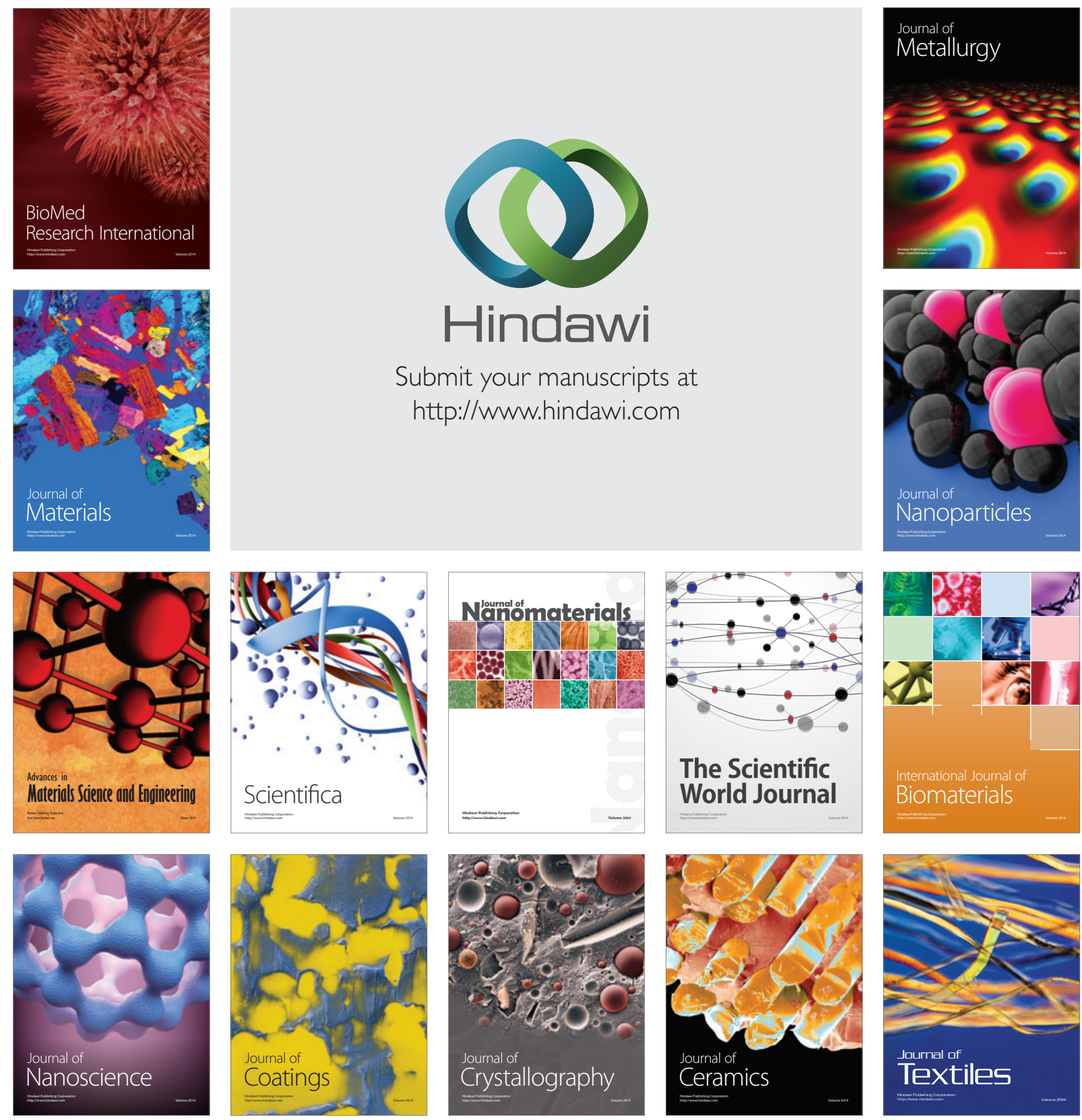